Voix et Images

voixetimages

\title{
Deux ouvrages fondamentaux
}

\section{Jacques Allard}

Volume 15, numéro 2 (44), hiver 1990

Pratiques illicites

URI : https://id.erudit.org/iderudit/200844ar

DOI : https://doi.org/10.7202/200844ar

Aller au sommaire du numéro

\section{Éditeur(s)}

Université du Québec à Montréal

\section{ISSN}

0318-9201 (imprimé)

1705-933X (numérique)

Découvrir la revue

\section{Citer cet article}

Allard, J. (1990). Deux ouvrages fondamentaux. Voix et Images, 15(2), 288-294. https://doi.org/10.7202/200844ar d'utilisation que vous pouvez consulter en ligne.

https://apropos.erudit.org/fr/usagers/politique-dutilisation/ 


\section{Recherches}

\section{Deux ouvrages fondamentaux}

\section{par Jacques Allard, Université du Québec à Montréal}

\section{Pour une nouvelle histoire littéraire}

La plus récente publication du Centre de recherche en littérature québécoise (CRELIQ) rappelle à tous les chercheurs du domaine le rôle moteur de ce regroupement de collègues de Laval. Né pour une bonne part de l'équipe du Dictionnaire des ceuvres littéraires du Québec, animée par M. Lemire de 1971 à 1987, le CRELIQ continue d'assurer le leadership du renouvellement des études. Après avoir délimité le corpus (avec le concours de quelque cinq cents collaborateurs), le groupe poursuit son travail sur la constitution de notre littérature, l'objectif général étant d'en arriver à produire une nouvelle histoire de notre littérature.

Or l'une des étapes décisive était bien de prendre en compte les acquis de la réflexion socio-historique depuis le déclin de la "nouvelle critique ". Il fallait aussi questionner les propositions déjà un peu canoniques, les grandes références du projet CRELIQ, celles de Even-Zohar, Habermas, Jauss, Bourdieu, Dubois, etc., et revenir sur les manuels et histoires littéraires en usage ici et ailleurs. C'est ce 
dont rend compte l'Histoire littéraire, théories, méthodes, pratiques ${ }^{1}$, fruit d'un colloque d'octobre 1986. L'ouvrage ne donne à lire que les textes présentés. En voici quelques aspects.

\section{L'histoire littéraire: celle d'un ensemble de paradigmes}

Clément Moisan, organisateur de la rencontre et éditeur, a d'abord fait place à la recherche de la scientificité de l'histoire littéraire: d'où ce premier tiers de l'ouvrage consacré aux "théories». Les concepts clés: le paradigme, le discours social, le système (ou polysystème). Pierre Ouellet ouvre le feu. Pour lui, à la suite de Jauss et Kuhn, c'est dans l'histoire des sciences qu'il faut aller chercher la notion fondatrice de "paradigme». Et

l'histoire littéraire n'est jamais que l'histoire des traits constitutifs d'un paradigme ou d'un ensemble de paradigmes différents, plus ou moins antagonistes, dont toute oeuvre est la reproduction. On ne lit les oeuvres qu'à l'aide des modèles dont elles sont, en quelque sorte, la projection... C'est à une histoire des modèles, donc des paradigmes, que la nature même de l'histoire, dans sa complexité, nous condamne...(p. 13-14)

C'est en utilisant pour sa part la notion de "paradigme moderne" que Lucie Robert ${ }^{2}$ conclut ensuite à la fétichisation historique du littéraire et à notre enfermement dans la logique du fétiche qui masque les événements et les pratiques qui ont présidé à sa naissance et à son institution. Elle propose donc de considérer le fétichisme lui-même comme objet de l'histoire littéraire, puisqu'il représente un moment important de l'objectivation de la littérature, bien qu'il soit la résultante d'une projection imaginaire sur le réel effectif (p. 23).

\section{Un système ouvert, un réseau, une hiérarchie multi-niveaux}

Clément Moisan prend le relai en réfléchissant sur "Lhistoire littéraire comme discours scientifiquen. Reprenant les propos de Greimas sur le relativisme inhérent aux théories sémiotiques ou linguistiques, phénomène attribuable à l'ensemble de concepts non définis qui sert de support aux sciences humaines, il déclare d'abord qu'il en va de même pour l'histoire littéraire. Et que l'on ne pourra arriver au faire et au savoir-faire scientifique qu'en mettant en place lidée de système. Après avoir établi la légitimité de sa démarche sur l'usage qu'en font Jauss, Genette, et Bourdieu après Saussure et Lanson (dont nous vivons encore), Moisan rejette le système fermé de l'histoire traditionnelle et retient le système ouvert, ou polysystème: système complexe qui implique la vie, le mouvement, l'échange, la relation... qui se fait réseau, hiérarchie à multiples niveaux. La discipline deviendrait ainsi

l'histoire de ce phénomène litteraire [...] de cette activité de production, de diffusion et de réception de textes par des individus qui sont euxmêmes conditionnés par leur être physique, social, politique et par leur histoire propre; l'histoire de ces textes qui sont situés dans un environnement social qui les investit (la société dans le texte) et où ils s'inscrivent (le texte dans la société)... (p. 28-29)

La fonctionnalité, l'intentionnalité et l'organicité du phénomène littéraire seraient ainsi analysées et évaluées. 
C'est en accord avec ces fondements d'une nouvelle histoire que Manon Brunet fait plus loin la critique des histoires traditionnelles, ou encore "sociologiques" récentes. L'histoire littéraire ne serait pas assez historienne, distanciée de son objet (trop proche de la critique), ne tiendrait pas suffisamment compte des "différences", au sens adapté de Saussure: une cuvre littéraire ne prend son sens que dans des séries littéraires (p. 38). Exemple: l'Influence d'un livre, quand on le met en rapport avec les faits politiques (1837-1838), on oublie l'apparition à cette époque d'une nouvelle esthétique. Font obstacle à la perception des "différences" le parti pris du chef-d'oeuvre, des genres majeurs (au mépris des mineurs), les fétichismes de l'écriture et de limprimé... Pour elle, la discipline renouvelée devrait observer la construction des différences, à partir d'un modèle de l'activité littéraire qui, dans toute la mesure du possible, lui permet de saisir toute l'étendue de la production des différences (p. 52).

\section{Priorité au social: l'interaction du littéraire et des autres discours}

Lintervention de Marc Angenot vient par après situer la problématique déjà ouverte. Non pas qu'il s'intéresse vraiment à la question. Elle lui paraîtrait plutôt réglée, si l'on se fie au postulat de sa propre recherche historique "en coupe synchronique». On sait que depuis plusieurs années, il étudie le discours social de l'année 1889 en France. Il résume donc ses perspectives de travail. Et partant, la place implicitement faite au discours littéraire et à son histoire. Définissant le discours social par la totalité de la production sociale $d u$ sens, il fait de l'expression littéraire une partie de l'interdiscursivité généralisée où se parlent la philosophie aussi bien que la chanson, la littérature novatrice autant que la nouvelle journalistique, etc. Dans cette perspective, la littérature joue le rôle du "fou du roi" des sociétés bourgeoises. Et son histoire relèverait d'une histoire globale du discours social. Ses propositions mettent donc de l'avant ce que Moisan appelait l'environnement social des textes, soumettant ces derniers au premier; elles étayent la dénonciation du fétichisme littéraire dont parlaient Robert et Brunet; elles "socialisent" l'histoire des paradigmes selon Ouellet. On peut en inférer que l'historien de la littérature, se définissant d'abord comme un historien du social et de l'idéologique, ne pourra faire l'histoire du littéraire qu'en le mettant en interaction avec tous les autres discours nobles ou triviaux avec lesquels il coexiste, délaissant le textocentrisme, lidéologie critique de l'art "pur ", subversif, mensonge fondateur de la pratique littéraire et du champ littéraire lui-même... On ne peut, selon Angenot, fonder le champ littéraire sur des exceptions, des inventeurs de langages, des logothètes comme Lautréamont, Joyce ou Kafka, et s'en servir comme alibi esthétique.

\section{Un discours de la formation du goût et du savoir-lire}

Faisant contraste avec les exposés précédents, Joseph Melançon clôt cette partie "Théories " en analysant le paradoxe de l'absence et de la présence du sujet qui traverse l'histoire littéraire (en France et au Québec) comme effet didactique. Pour conclure qu'en littérature, l'objet convoité, l'objet / valeur, échappe au savoir et que la didactique du goat rejoint mal la didactique de la connaissance littéraire, particulièrement au XIX ${ }^{\mathrm{e}}$ siècle. $\mathrm{Au} \mathrm{XX}$, cette dialectique du goût et du savoir serait occultée, les enseignants ne prétendant plus assurer maintenant la 
formation du goat dans leur transmission d'un savoir-lire, de leur tradition de lecture.

\section{Méthodes}

Après avoir rapporté plus en détail la partie "théorique», je passe plus rapidement sur le reste de l'ouvrage qui n'en est pas pour autant moins riche. Sous la rubrique "Méthodes", on trouve d'abord les contributions: rapide, d'Alain Viala, étoffée de Laurent Mailhot sur les problèmes de la périodisation. Le premier passe par la notion de champ littéraire afin de contester le découpage en tranches séculaires et la périodisation de type politique ou événementielle. S'appuyant sur des changements de configurations et de "dispositifs" propres au champ, il propose son hypothèse des sept phases de l'histoire du champ littéraire français, de 1630 à aujourd'hui: constitution (1630-1665), consolidation (16651750), mutation (1750-1830), consécration (1830-1850), expansion (1850-1914), exploitation (1914-1950?), redistribution (1950?- ). Le second, sur la même longueur d'ondes, passe en revue différentes facettes de son sujet: comme on le sait, Laurent Mailhot prépare une histoire littéraire qu'il veut distinguer d'ailleurs d'une "histoire de la littérature". Pour lui aussi, l'histoire littéraire ne peut être que sociale, culturelle (p. 107). Il remet en question plusieurs usages faits, ici et ailleurs, de notions clés: générations, milieux, périodes, ruptures, styles, etc. Il isole des problèmes spécifiques: la Nouvelle-France québécoise, le Mouvement littéraire et patriotique de Québec, les tendances récentes (formalisme, féminisme, marxisme, américanisme).

Roger Fayolle s'interroge ensuite: Nous sommes assez savants en littérature, mais le sommes-nous assez en histoire...? [Cette activité d'historien littéraire] n'est-elle pas souvent étroitement conditionnée par le milieu socio-politique...? pour finalement en appeler au regroupement des historiens dans une réécriture de l'histoire de la littérature française. Quant à Jacques Michon, évoquant au passage les recherches de son groupe sherbrookois sur les formes narratives et discursives (du roman québécois) légitimées par la critique ou encore ses travaux sur l'édition, il rappelle que pour lui, l'histoire littéraire consiste à étudier l'évolution des rapports entre certaines formes discursives et les stratégies ou les parcours sociaux des agents qui les véhiculent ou les diffusent, en conformité avec une sociologie des milieux intellectuels et une théorie de la réception (p. 142). Il donne l'exemple du poète exotiste Paul Morin qui fut aussi un historien de la littérature (vers 1910), à l'époque terroiriste où Mgr Camille Roy pratiquait une critique d'édification. Morin ne serait suivi sur cette voie que trente ans plus tard avec l'édition critique de Nelligan préparée par Luc Lacoursière.

De son côté, Giuliana Bertoni del Guercio (Centro Europeo Dell'Educazione, Frascati, Roma) rend compte d'une recherche faite à son centre, où il apparaît que les enseignants du secondaire refusent les manuels et anthologies existants sans encore savoir comment les remplacer. Paul Wyczynski défend ensuite la nécessité des biographies, en définit les objectifs et les grands principes, s'appuyant sur les apports récents des éditions critiques de la Scouine et de l'œuvre de F.X. Garneau. Puis Milan V. Dimic propose le modèle polysystémique de Even-Zohar parmi les méthodes utiles pour les études canadiennes comparées, 
tout en déplorant que le corpus ne soit pas complètement établi, ni suffisamment exploré (p. 189).

\section{Pratiques}

Après ces questionnements aussi divers que stimulants, viennent les "Pratiques". Antoine Compagnon soumet une réflexion sur le retour d'un souci historique après la nouvelle critique. Il s'agit pour lui d'un retour à Lanson et particulièrement à Brunetière dont il fait les précurseurs, sinon les ancêtres de Jauss et de la théorie de la réception. Ainsi la théorie des genres de Brunetière était déjà une esthétique de la réception (p. 211-212). Là-dessus, Brian T. Fitch pose un problème peu fréquent: la double réception faite à l'œuvre bilingue de Samuel Beckett. Le paradoxe, non résolu: les cultures anglaise et française contemporaines intègrent naturellement à leur patrimoine l'Innommable/The Unnamable et Compagnie/Company, leur faisant côtoyer aussi bien À la recherche du temps perdu et la Route des Flandres que Finnegans Wake et The Waves.

La contribution d'Éva Kushner porte sur un exemple beaucoup plus large, celui de la Renaissance. Elle-même engagée dans la publication d'une Histoire comparée des littératures de langues européennes, pour la partie concernant l'époque 1400-1610, elle montre comment s'est déconstruite et reconstruite la discipline. Dorénavant, la "littérature" est une notion très extensive: jamais dissociée du socio-historique, elle inclut plus que le consacré, allant jusqu'aux écrits populaires, didactiques, religieux, savants, etc., ce qui confirme le mouvement global contemporain d'une "sociologisation" de l'entreprise historicolittéraire.

Trois autres cas sont enfin présentés qui concernent le domaine strictement québécois. Denis Saint-Jacques (directeur du CRELIQ) discute du grand projet collectif: "Histoire des processus de légitimation et d'autonomisation de la littérature québécoise dans sa production, son discours et sa réception". Deux termes stratégiques sont ici portés en titre de son exposé: "Nationalisation et autonomisation " qui renvoient à sa réflexion "exploratoire ". Comme il le dit lui-même: $S i$ jai voulu m'arrêter sur ces deux postulats de l'histoire littéraire - socialement la litterature existe et elle est nationale -, c'est bien entendu afin d'en marquer à la fois linterdépendance et la relative contrariété. Maurice Lemire décrit de son côté le fonctionnement de l'«instance critique" au XIX ${ }^{e}$ siècle. On assiste selon lui à deux mouvements: l'un qui concerne l'autonomisation de la critique par rapport aux autres pouvoirs et l'autre qui cherche à instaurer un système de contrôle des lectures, deux faces d'une même politique qui a été, comme on le sait, cléricale, ce qui a donné des verdicts plutôt qu'une critique littéraire. René Dionne ressort là-dessus le texte canonique "Le mouvement littéraire en Canada" de l'abbé et propagandiste de la foi littéraire Henri-Raymond Casgrain. Il démontre que dans ce texte, Casgrain n'a fait que reprendre (en 1866) le discours conservateur, patriotique, messianique qui s'écrit de James Huston (1848) à Edme Rameau de Saint-Père (1859).

À la fin de sa présentation de l'ouvrage, Clément Moisan dit que cet ensemble de textes [...] constituent une contribution majeure à la réflexion sur notre 
discipline (p. X). On reconnaîtra volontiers l'ampleur de ce panorama de réflexions sur le mouvement actuellement le plus important dans les études littéraires. On pourra pourtant s'étonner d'une presque absence de débat dans ce colloque qui consacre la nouvelle vigueur de la sociologie: les intervenants ne discutent que très rarement les références communes, tout en étant généralement d'accord pour enterrer les mouvements précédents (la nouvelle critique, le structuralisme).

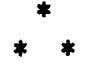

Tous ceux qui, comme Milan Dimic et l'équipe de l'Université de l'Alberta (travaillant sur l'histoire de linstitution littéraire au Canada), souhaitent la progression des études comparées canado-québécoises trouveront très utiles ces "Cahiers de littérature comparée. Le premier numéro ${ }^{3}$ comprend, outre la bibliographie elle-même, une brève histoire de la discipline au Canada par David M. Hayne et un « Bilan de la recherche * par Antoine Sirois.

Comportant plus de mille titres, la bibliographie bilingue (comme le reste du numéro) prend la suite de celle qui a paru annuellement depuis 1976 dans la Revue canadienne de littérature comparée, conservant grasso modo les catégories déjà utilisées, en les enrichissant. Ce qui donne les rubriques suivantes: bibliographies et répertoires; ouvrages de référence; anthologies et recueils; périodiques courants; études générales ou comparatives; comparaison d'auteurs (peu répandue) et d'ouvrages, de thèmes (la nature, le père, la mère, le héros, le prêtre, le ministre, le soldat; les mouvements sociaux: nationalisme, séparatisme, racisme...) et d'influences (pas de l'une sur l'autre: anglophone et francophone s'ignorent réciproquement), de genres (le roman l'emporte) et de formes; épistémologie et méthodologie; l'institution littéraire; et les articles généraux. On trouve aussi dans l'ouvrage un index des auteurs étudiés (prédominent: Aquin, Atwood, Cohen, Godbout, Grove, Hébert A, Langevin, Laurence, MacLennan, Richler, Ringuet, Roy G.) et un autre des auteurs d'ouvrages et d'études. Enfin, la liste des périodiques utilisés.

Cet ouvrage, modeste dans sa réalisation éditoriale, rappelle opportunément l'engagement assumé depuis vingt-cinq ans par le Département des lettres et communications de l'Université de Sherbrooke dans le domaine de la « littérature canadienne comparée». On notera que cette dernière expression ne renvoie plus aux seules littératures francophone et anglophone: elle inclut aussi les nouvelles manifestations allophones au Canada.

1 Clément Moisan (sous la direction de), l'Histoire littéraire, théories, méthodes, pratiques, Québec, les Presses de l'université Laval, 1989, 284 p.

2 Voir son ouvrage tout récent l'Institution du littéraire au Québec, Québec, les Presses de l'université Laval, 1989, 272 p. (CRELIQ/Vie des lettres québécoises). Après avoir clairement établi les fondements théoriques de sa démarche, la directrice de notre revue étudie comment la valeur littéraire a été historiquement établie depuis le début du XIX siècle jusqu'après la Seconde Guerre mondiale. Sa description précise de la vie du littéraire pose les jalons d'une 


\section{VOIX \& IMAGES / 44, hiver 1990}

nouvelle histoire littéraire du Québec: de la naissance de l'imprimé jusqu'au a fétichisme littéraire ", en passant par limprimeur, l'éditeur, le libraire, l'écrivain, le public, l'action sociale, «La France et nous *, l'académisme, la modernité, etc.

3 Antoine Sirois, Jean Vigneault, Maria van Sundert et David Hayne, Bibliographie d'études de littérature canadienne comparée 1930-1987, Sherbrooke, Université de Sherbrooke, Département des lettres et communications, section d'études anglaises, $130 \mathrm{p}$. (Cahiers de littérature canadienne comparee, $n^{0} 1$ ). 